\title{
Signal of single scattering albedo in water leaving polarization
}

\author{
J. Piskozub \\ piskozub@iopan.gda.pl \\ W. Freda
}

\author{
Institute of Oceanology, Polish Academy of Sciences, ul. Powstańców Warszawy 55, 81-712, Sopot, \\ Poland \\ Department of Physics, Gdynia Maritime University, ul. Morska 81-87, 81-225, Gdynia, Poland
}

The purpose of this article is to examine whether the change in single scattering albedo in the surface layer of the sea will cause significant changes in the polarization of light emerging from the sea. The results of a polarization resolving Monte Carlo radiative transfer calculations are presented. Bubble clouds of different bubble concentrations are used to achieve a wide range of single scattering albedo variability. The variability of the polarization signal is largest in the direction looking towards the sun which is unfortunate due to sun reflection. However the variability is also significant at direction perpendicular to solar azimuth angle which should it make possible to use this signal in remote sensing. The polarization degree of water leaving radiation, together with reflectance can be used to determine the backscattering ratio of the observed sea water.

[DOI: http://dx.doi.org/10.2971/jeos.2013.13055]

Keywords: Oceanic light scattering, degree of polarization, single scattering albedo

\section{INTRODUCTION}

Both the polarization of the upward radiance that leaves the sea surface, as well as polarization of the radiance reflected from the surface are the important sources of information on the presence and concentration of so called water constituents and the sea surface slopes. This information, is especially important for the study of phenomena associated with the seaatmosphere boundary (breaking waves, bubble clouds, etc.) and till now has not been practically used. In the literature we were able to find the only one Monte Carlo algorithm to solve the radiation transfer equation in the sea, which contains the polarization. Unfortunately only two theoretical works using this program were created by its author [1, 2]. And another only one work [3], comparing the results of the Monte Carlo code containing the polarization and the measured data was published so far. It seemed expedient to create the second independent program of its kind in the world, specifically targeted to study phenomena related to surface of the sea. Different approach to model the water constituents impact on the polarization of water leaving radiance was shown by [4] and later by [5]. They developed the radiative transfer code (called OSOA) to approximate the radiance and the degree of polarization in the ocean-atmosphere environment. Their study about phytoplankton and mineral suspensions shown that water leaving degree of polarization depend strongly on concentration of these water constituents and the zenith angle of water leaving radiance.

\section{METHODS}

A new version of Monte Carlo code, previously used for almost 20 years to solve problems of ocean optics [6, 7] and im- prove measurement error correction schemes [8]-[10], was developed which includes the polarization taking into account both light scattering Mueller matrices of seawater and polarization effects associated with the waved sea surface. The program allows to track changes of the full Stokes vector for each virtual "photon" for any of the Mueller scattering matrix. For a radiation transmission through the sea surface radiation and for a reflection the Mueller matrices corresponding to the Fresnel formulas were used [11]. The angles used in the Mueller matrices associated to rotation of the coordinate system needed to adapt the Stokes vectors were enumerated using the formula of sines for spherical triangles following [12].

As a first step the all of non-trivial elements of the Mueller matrix for the bubble size distributions (measured acoustically at the Scripps Institution of Oceanography, see $[13,14])$ were calculated. We have used a normalized form of the Mueller matrix (relative to the element $S_{11}$ ), which therefore by definition equal to unity $\left(S_{11}=1\right)$. The Mie theory shows that the only other non-zero matrix elements are $S_{22}=1$ (since $S_{22}=S_{11}$ ), $S_{12}=S_{21}, S_{33}=S_{44}$ and $S_{34}=-S_{43}$ (actually only 3 of these 4 are independent, but the calculations do not use this fact).

Non-trivial elements of the scattering matrix $S_{12}, S_{33}$ and $S_{43}$, calculated with the Mie theory, are shown in the Figure 1, as a function of scattering angle for size distribution of bubbles "bub43" (the average distribution for the amount of air in water specified by void fraction between $10^{-4}$ and $10^{-3}$ ).

The calculations showed that the Mueller matrices have practically the same functional form for all considered size distri- 


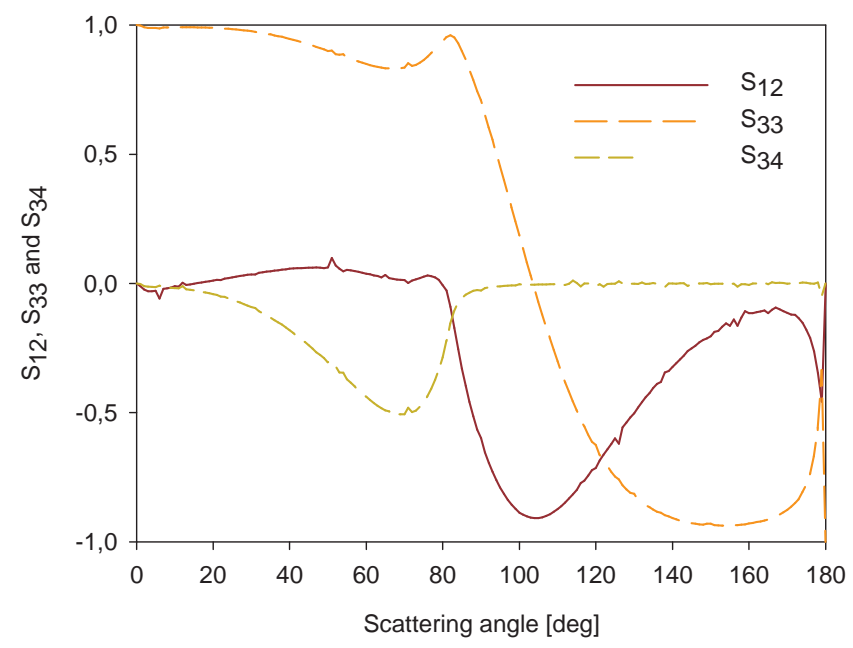

FIG. 1 Calculated elements of the light scattering Mueller matrix for the size distribution of bubbles "bub43" (see text for details).

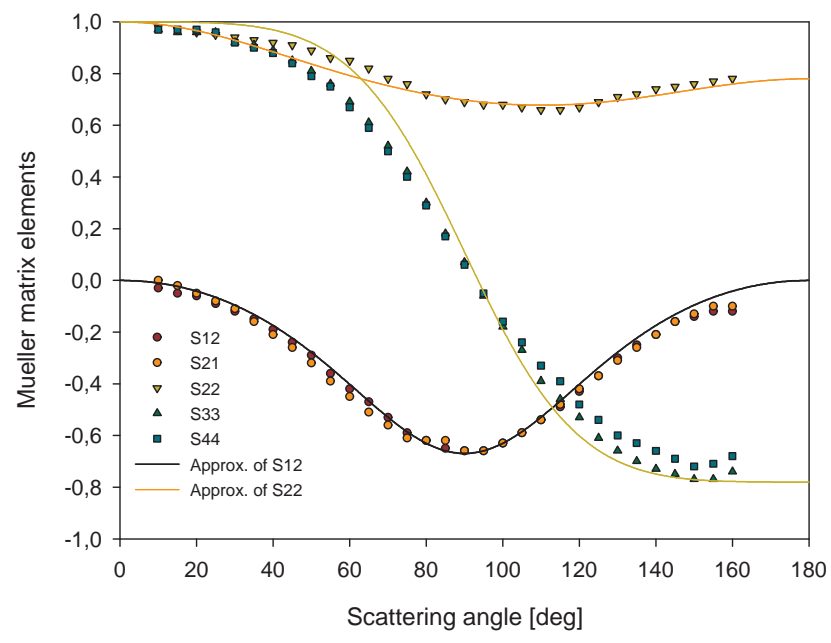

FIG. 2 Mean values of the Mueller matrix elements measurements by [15], in the Atlantic and Pacific (points) and their analytical approximation (lines).

butions and for different void fractions, that is why for further calculations only the "bub43" size distribution was used.

For the pure water the well-known Mueller matrix of Rayleigh scattering can be used. However Mueller matrices of suspended particulate matter are not known as an analytic formulas.

Practically the only available data on them are experimental results presented by [15] measured in the waters of the Atlantic and Pacific and not widely published data collected by the author [16], (unfortunately only the elements $S_{12}, S_{21}$ and $S_{22}$ measured in coastal waters in Miami, Florida). Measured functions differ from those obtained from Mie theory, the value of measured element $S_{22}$ (depolarization) is lower than 1 and values of the $S_{34}$ and $S_{43}$ elements are practically zero. Since the measurements of Voss and Fry are more representative, we have used their tabulated values to create a rough analytical form, which was necessary for the further modeling (see Figure 2).
We have found an approximated analytical elements of the Mueller matrix (for the scattering angles $\theta$ in degrees) measurements of both Voss and Fry results and recent by one of us for coastal waters [16]. The parameterization has only one free parameter $A$ ( $A=0.11$ in the case of Voss and Fry results), unlike a previously published 4-parameter one [17]. The new parameterization has the following analytical form:

$$
\begin{aligned}
S_{12}= & (1-3 A) \frac{\cos ^{2}(\theta)-1}{\cos ^{2}(\theta)+1} \\
S_{33}= & (1-A) \frac{2 \cos (\theta)}{\cos ^{2}(\theta)+1}+A \\
S_{22}= & (1-A)+A \cos (\theta)-1.75 \cdot A \cdot \sin ^{2}(\theta) \\
& -0.1 \cdot A \cdot \sin ^{2}(-2 \theta) .
\end{aligned}
$$

\section{RESULTS AND DISCUSSION}

The above mentioned parametrized form of Mueller matrix elements was used as an input data to the Monte Carlo program, that has been adapted for the calculations of polarization in the Stokes vector format. The modeling was carried out to check whether the change in concentration of bubbles in the surface layer of the sea will result in significant changes in the polarization of light emerging from the sea, that could allow remote sensing of clouds of bubbles by polarization.

In the first tests layers of bubbles of various densities were used (here the parameter is the scattering coefficient $b_{b u b}=0,1,2,4$ and $8 \mathrm{~m}^{-1}$ associated with the corresponding phase functions and Mueller matrices calculated from the Mie theory). The others parameters were invariable, namely the absorption coefficient $a=0.4 \mathrm{~m}^{-1}$, the scattering coefficient of clean seawater $b_{w}=0.2 \mathrm{~m}^{-1}$ and the particle scattering coefficient $b_{p}=0.8 \mathrm{~m}^{-1}$ for which the Petzold phase function [18] and the Mueller matrix of [15] were used. Thus the single scattering albedo, defined as:

$$
\omega_{0}=\frac{b_{w}+b_{p}+b_{b u b}}{a+b_{w}+b_{p}+b_{b u b}},
$$

varies from 0.71 to 0.96 . Ocean was illuminated by the sun of zenith angle 30 degrees with black sky (which for simplicity of modeled phenomenon is a typical approach in an optics model) and a flat sea surface (for the same reasons).

The Degree of Polarization $(D O P)$ is by definition, equal to:

$$
D O P=\frac{\sqrt{Q^{2}+U^{2}+V^{2}}}{I},
$$

where $[I, Q, U, V]$ is the Stokes vector.

In the Figures 3 and 4 the DOP of water leaving radiation was shown for the two vertical planes which are perpendicular to each other. The effects of reflection were not present because of the assumed black sky and flat sea surface. In the Figure 3 the observation plane is parallel to the incident rays (the sun is on the left) and in the Figure 4 the observation plane is perpendicular to the rays of the sun (sun is behind or in front of the observation plane). 


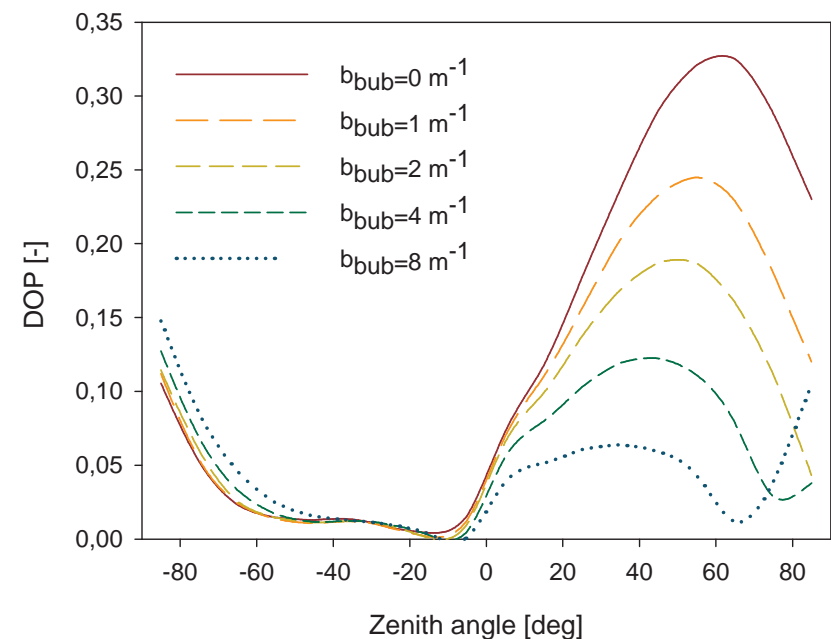

FIC. 3 The average value of the DOP for the sea illuminated by a light source of $30 \mathrm{deg}$ zenith angle measured at different zenith angles at a vertical plane parallel to incident radiance (the modeled "light source" is on the left side of the figure). Positive and negative values of the zenith angle should be treated conventionally: means the same angle but on opposite sides of the zenith.

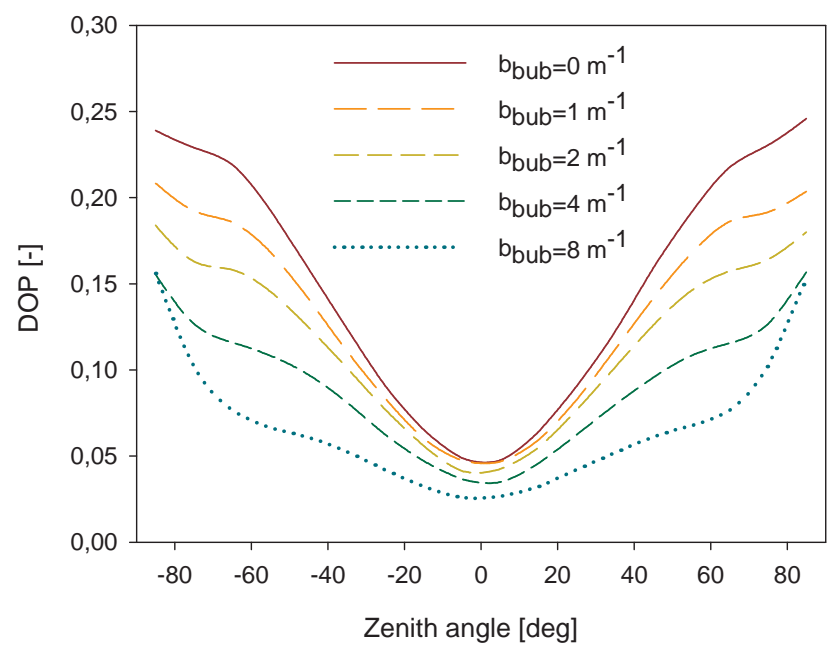

FIG. 4 The average value of the DOP for the sea illuminated by a light source of $30 \mathrm{deg}$ zenith angle measured at different zenith angles at a vertical plane perpendicular to incident radiance (the modeled light source is "behind" the viewer). Positive and negative values of the zenith angle should be treated conventionally: means the same angle but on opposite sides of the zenith.

The results show that a highly scattering bubble cloud layer causes a significant depolarization of water leaving radiation. The Figure 3 shows that the smallest value of polarization (and the least variation with the concentration of bubbles) occurs for single scattering into backward direction, (the direction of the sun). While at the opposite side the polarization values depends strongly on the scattering coefficient associated with the bubbles. However, this is the angle zone of the sun reflection from the surface (sun blinks), that is why it is important that this variation occurs also at the observation plane perpendicular to the sun (Figure 4), especially around zenith angles 50 to 60 degrees. This should make it possible to develop a remote sensing algorithm based on observa-

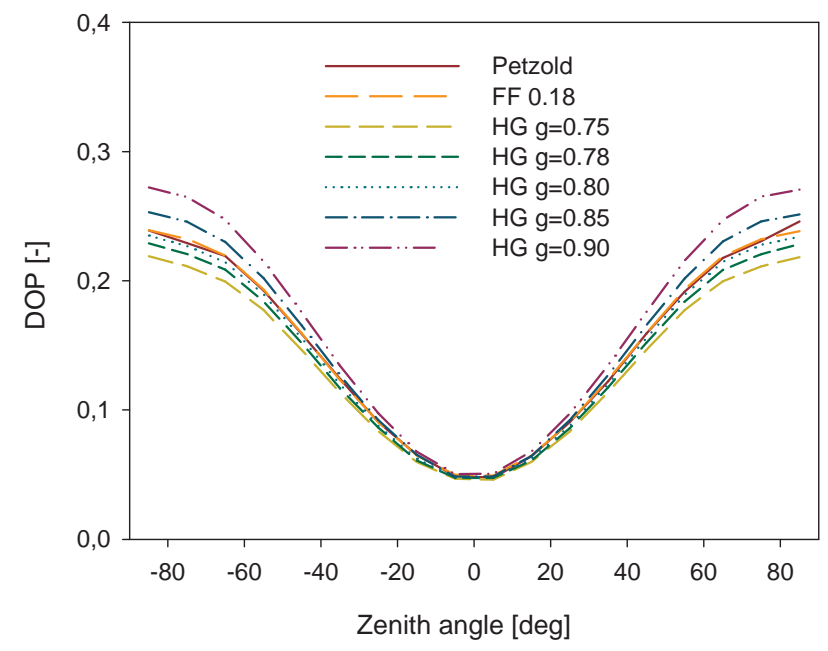

FIG. 5 The average value of the $D O P$ for the sea illuminated by a light source of $30 \mathrm{deg}$ zenith angle measured at different zenith angles at a vertical plane perpendicular to incident radiance (the modeled light source is "behind" the viewer). Positive and negative values of the zenith angle should be treated conventionally: means the same angle but on opposite sides of the zenith.

tions done at a non-zero zenith angle and azimuth angle about 90 deg from the sun.

A similar pattern of variation of degree of linear polarization for two perpendicular planes was presented by [19]. Although their results have been obtained by another method, and show variation of the linear (not total) polarization degree just below sea surface, the results confirm the dependence of the polarization of light on the propagation direction (relative to the sun) and the inherent optical properties of the sea.

Because the depolarization of water leaving radiance seems strongly dependent on scattering, we put forward a hypothesis that the controlling factor is single scattering albedo $\omega_{0}=b /(a+b)$. In real ocean all remotely sensed properties are depth averaged with the surface waters best represented in the water leaving signal [20]. For simplicity we will concentrate on the ideal case of an optically homogeneous ocean of infinite depth. In such a case, absolute values of absorption and scattering are not important as rescaling them both (which is equivalent to rescaling the length unit) changes nothing in an infinitely deep water body. All that is needed to calculate the volume of water leaving radiance in such a case is the ratio of scattering to absorption and the scattering phase function. However because depolarization is caused by scattering, one can expect that it is related to the average number of scattering events per photon. This parameter is directly dependent on by single scattering albedo (known also for a reason photon survival probability).

To test of the hypothesis on $\omega_{0}$ being the controlling parameter for water leaving polarization, we checked its dependence on the other parameter it can depend on in the case of homogeneous infinite ocean, namely the phase function. Figure 5 shows the results for the plane perpendicular to solar azimuth angle. We used average Petzold phase function [18], FournierForand phase function [21] most similar to the Petzold one 
using parametrization of [22] and several Henyey-Greenstein phase functions [23] of different asymmetry parameter $g$.

The results show that although water leaving polarization depends on phase function the dependence is much weaker than for single scattering albedo. The range of Henyey-Greenstein functions chosen with asymmetry parameter $g$ from 0.75 to 0.9 is much wider than one for natural waters. Interestingly, using phase functions of similar backscattering ratios $\left(b_{b} / b\right)$, namely Petzold, Fournier-Forand and Henyey-Greenstein for $g=0.78$ and $g=0.8$ results in almost identical values of water leaving polarization. This is important because the limited available observational data seem to suggest that at least locally, backscattering ratios seem to have little variability $[24,25]$. In fact the water leaving polarization values for different phase functions of identical backscattering ratios seem to be even more similar than reflectance values also modeled for different phase functions of identical $b_{b} / b$, considered by [26].

However, even if the dependence of water leaving radiance on both single scattering albedo (equal to $b /(a+b)$ ) and backscattering ratio (equal to $b_{b} / b$ ) needed to be taken into account, using the additional information from the volume of water leaving radiance (or more exactly reflectance which is proportional to $b_{b} / a$ ) should make it possible to inverse the problem. This is because we have two independent observables (reflectance and polarization) and only two independent parameters to calculate because it is easy to show that:

$$
\frac{b_{b}}{b}=\frac{b_{b}}{a} \frac{1-\omega_{0}}{\omega_{0}}
$$

This is why we believe that the degree of polarization of water leaving radiation should be used to augment reflectance in remote sensing of natural waters.

\section{CONCLUSION AND FINAL REMARKS}

We have shown using Monte Carlo radiative transfer modeling that water leaving radiation carries information about single scattering albedo of sea water. The modeling results suggest that the optimal way to record the polarization variability is from a tilted direction approximately $90 \mathrm{deg}$ from solar azimuth angle. The polarization signal is also dependent on the backscattering ration of the average scattering phase function though weaker than on single scattering albedo. In any case, even if the phase function dependence cannot be neglected, it should still be possible to retrieve $\omega_{0}, b_{b} / b$ and $b_{b} / a$ values from polarization and reflectance data only thanks to the algebraic relations between the three variables. The method should be especially good at detecting $\omega_{0}$ caused by the presence of bubble clouds but should be sensitive also to its variability caused by other sea water constituents. It seems possible to develop such a remote measurement method, at least from ships and aircraft, but also possibly satellite (although not in the usually preferred vertical direction). Perhaps it would be optimal to use the relationship of the polarization reflectance signal measured for different zenith angles. Further Monte Carlo tests are needed involving other zenith angles of the sun and multiple sets of sea water inherent optical proper- ties, especially ones with realistic sky light and therefore additional polarization of the surface reflected irradiance. Repeating some of the tests using another, independently developed, polarization aware Monte Carlo code would be helpful validation of our results. However, the most important step to such procedure should be developing and implementing instruments for routine measurements of water leaving polarization.

\section{ACKNOWLEDGEMENTS}

We are indebted to Ken Voss for both inspiration and critical comments. This paper was carried out within the framework of the SatBałtyk project funded by the European Union through European Regional Development Fund, (contract No. POIG.01.01.02-22-011/09 entitled "The Satellite Monitoring of the Baltic Sea Environment"). Moreover W. Freda was supported by grant No. UMO-2012/07/D/ST10/02865 received from National Science Centre of Poland. The partial support for this study was also provided by statutory research tasks in IO PAS, Sopot.

\section{References}

[1] G. W. Kattawar and C. N. Adams, "Stokes Vector Calculations of the Submarine Light Field in an Atmosphere- Ocean with Scattering According to a Rayleigh Phase Matrix: Effect of Interface Refractive Index on Radiance and Polarization," Limnol. Oceanogr. 34, 14531472 (1989).

[2] C. N. Adams and G. W. Kattawar, "Effect of volume-scattering function on the errors induced when polarization is neglected in radiance calculations in an atmosphere-ocean system," Appl. 0pt. 32, 4610-4617 (1993).

[3] J. T. Adams, E. Aas, N. K. Hjøerslev, and B. Lundgren, "Comparison of radiance and polarization values observed in the Mediterranean Sea and simulated in a Monte Carlo model," Appl. Opt. 41, 2724-2733 (2002).

[4] M. Chami, R. Santer, and E. Dilligeard, "Radiative Transfer Model for the Computation of Radiance and Polarization in an OceanAtmosphere System: Polarization Properties of Suspended Matter for Remote Sensing," Appl. Opt. 40, 2398-2416 (2001).

[5] M. Chami, "Importance of the polarization in the retrieval of oceanic constituents from the remote sensing reflectance," J. Geophys. Res. 112, C05026 (2007).

[6] P. Flatau, J. Piskozub, and J. R. Zaneveld, "Asymptotic light field in the presence of a bubble-layer," Opt. Express 5, 120-124 (1999).

[7] J. Piskozub and D. McKee, "Effective scattering phase functions for the multiple scattering regime," Opt. Express 19, 4786-4794 (2011).

[8] J. Piskozub, "Effects of surface waves and sea-bottom on selfshading on in-water optical instruments," Proc. SPIE 2258, 300-308 (1994).

[9] J. Piskozub, A. R. Weeks, J. N. Schwarz, and I. S. Robinson, "SelfShading of Upwelling Irradiance for an Instrument with Sensors on a Sidearm," Appl. 0pt. 39, 1872-1878 (2000),

[10] D. McKee, J. Piskozub, and I. Brown, "Scattering error corrections for in situ absorption and attenuation measurements," Opt. Express $16,19480-19492$ (2008). 
[11] D. Miyazaki, N. Takashima, A. Yoshida, E. Harashima, and K. Ikeuchi, "Polarization-based Shape Estimation of Transparent objects by Using Raytracing and PLZT Camera," Proc. SPIE 5888, 1-14 (2005).

[12] A. D. Code, and B. A. Whitney, "Polarization from scattering in blobs," Astrophys. J. 441, 400-407 (1995).

[13] J. Piskozub, D. Stramski, E. Terrill, and W. K. Melville, "Influence of Forward and Multiple Light Scatter on the Measurement of Beam Attenuation in Highly Scattering Marine Environments," Appl. Opt. 43, 4723-4731 (2004).

[14] J. Piskozub, D. Stramski, E. Terrill, and W. K. Melville, "Small-scale effects of underwater bubble clouds on ocean reflectance: $3-D$ modeling results," Opt. Express 17, 11747-11752 (2009).

[15] K. J. Voss and E. S. Fry, "Measurement of the Mueller matrix for ocean water," Appl. 0pt. 23, 4427-4439 (1984).

[16] W. Freda, "Angular variability in the depolarization of scattered light," Zesz. Nauk. Akademii Morskiej w Gdyni 60, 119-127 (2009), (in Polish).

[17] A. A. Kokhanovsky, "Parameterization of the Mueller matrix of oceanic waters," J. Geophys. Res. 108, 3175 (2003).

[18] T. J. Petzold, "Volume scattering functions for selected ocean waters," in Tech. Rep. SI0, 72-78 (Scripps Institution of Oceanography, San Diego, 1972).
[19] A. Ibrahim, A. Gilerson, T. Harmel, A. Tonizzo, J. Chowdhary, and S. Ahmed, "The relationship between upwelling underwater polarization and attenuation/absorption ratio," Opt. Express 20, 2566225680 (2012).

[20] J. Piskozub, T. Neumann, and L. Wozniak, "Ocean color remote sensing: choosing the correct depth weighting function," Opt. Express $16,14683-14688$ (2008).

[21] G. Fournier and J. L. Forand, "Analytic phase function for ocean water," Proc. SPIE 2258, 194-201 (1994).

[22] C. D. Mobley, L. K. Sundman, and E. Boss, "Phase Function Effects on Oceanic Light Fields," Appl. Opt. 41, 1035-1050 (2002).

[23] L. C. Henyey and J. L. Greenstein, "Diffuse radiation in the galaxy," Astrophys. J. 93, 70-83 (1941).

[24] W. Freda, T. Król, 0. V. Martynov, E. B. Shybanov, and R. Hapter, "Measurements of Scattering Function of sea water in Southern Baltic," Eur. Phys. J.-Spec. Top. 144, 147-154 (2007).

[25] W. Freda and J. Piskozub, "Improved method of Fournier-Forand marine phase function parameterization," Opt. Express 15, 1276312768 (2007).

[26] W. Freda, and J. Piskozub, "Revisiting the role of oceanic phase function in remote sensing reflectance," Oceanologia 54, 29-38 (2012). 